\title{
The design of a new type of pipe rack for civil construction
}

\author{
Wu Qingbo ${ }^{1 *}$, Xiao Yinan $^{1}$, Chen Qiuling ${ }^{1}$ \\ ${ }^{1}$ Wuhan University of Technology, School of civil ENGINEERING and ARCHITECTURE
}

\begin{abstract}
At present, the traditional way of placing building pipe fittings has many problems, such as labor, large space occupation, and insecurity, while the shelves on the market are not easy to remove the high pipe fittings and have low efficiency. In this paper, a new type of pipe laying frame is proposed, which consists of adjusting mechanism, taking mechanism and resetting mechanism. It not only optimizes the defects of the common display rack in the market, but also innovates the fixation and arrangement of the internal pipe fittings. It can not only solve the problems existing in the existing building pipe fitting display rack technology, but also reduce the problems and disadvantages in the use process. Under the actual application, it can improve the placement efficiency of the pipe fittings and reduce the staff, so as to improve the practicability of the rack.
\end{abstract}

\section{Introduction}

After the reform and opening up, China's urbanization process has achieved rapid development. The urbanization rate has grown from $17.9 \%$ at the beginning of the reform and opening up to $54.77 \%$ in 2014 [1]. In the process of China's economic development and urbanization, the construction of public and civil buildings and other facilities in the construction industry is indispensable. As of February 2019, with the development of construction projects, taking Wuhan as an example, there are more than 5400 construction projects. According to the statistics of relevant data, 9 NEW projects are added every day on average, while in the whole country, on average, every day More than 100 new projects have been added, and the construction site has become a scenic spot everywhere in the city.

Civil construction pipe fitting is a common material on the construction site, which has a very good application in the project, especially in the construction of hydropower, heating and ventilation, often need to use a lot of plumbing pipes. Building pipe fittings have the characteristics of large amount of application, large land occupation and difficult to store. At present, most of the traditional steel pipes or pipes are built with square wood on the ground first. Because the pipe fittings are generally round pipes, the upper wood can only be stacked into a cone with less upper wood and more lower wood, which not only occupies a lot of construction ground, but also reduces the construction site It is easy to fall, causing safety hazards to workers. In Duan Hongde's "how to avoid the waste of Construction Engineering" article, it is mentioned that one of the waste status and outstanding performance of the project construction site is that the improper storage and use of construction raw materials lead to the fearless loss of construction raw materials [2]. In this design, by adjusting the mechanism, the pipe can be placed in a rectangle with high utilization rate of the site; the cooperation of the take and reset mechanism can not only properly keep the pipe, but also reduce the loss of the pipe in the process of pick-and-place.

\section{INTRODUCTION TO OVERALL DESIGN}

In this design, the pipe fitting adjusting mechanism, pipe fitting taking mechanism and device reset mechanism are integrated into a design scheme[3]. The pipe fitting adjusting mechanism is used to receive the pipe fittings from transportation, and through the operation of the adjusting mechanism of the designed device, the pipe fittings of the received device are arranged in space, so that the pipe fittings can be arranged orderly at the place where the device holds the pipe fittings, and it is conducive to the operation of the pushing device in the pipe fitting taking mechanism, and the pipe fittings are pushed into the taking groove; the pipe fitting taking mechanism is driven With the cooperation of the device, the lifting groove and the buffer device, the pipe fittings pushed into the limit baffle and the buffer support plate are transported in the vertical direction, so that the pipe fittings are arranged in a single row of vertical space, so as to facilitate the user to take the pipe fittings more effectively and conveniently; the reset mechanism of the device unlocks the interlocking locking hook through the foot pedal, so that the buffer support plate and the limit baffle are vertically upward Movement, return to the initial position, re receive the pipe fittings pushed into the groove by the push device of the adjustment mechanism, and carry out a new round of work cycle. The system design diagram is shown in Figure 1 below:.

\footnotetext{
* Corresponding author: author@e-mail.org
} 


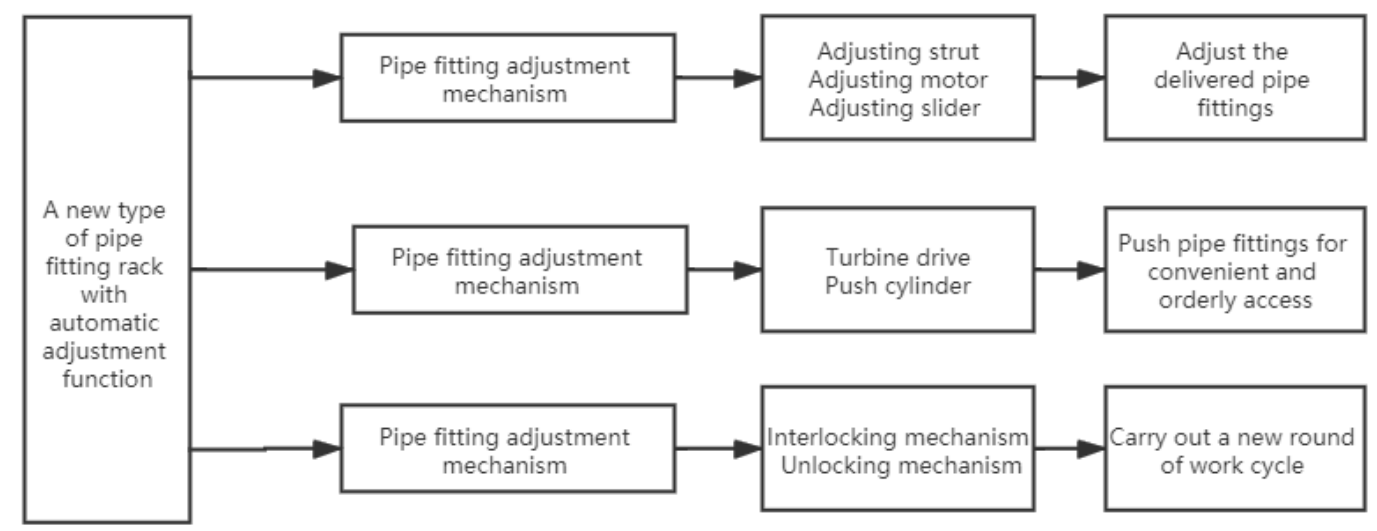

Fig. 1 Composition diagram of a new type of pipe fitting system with automatic adjustment function

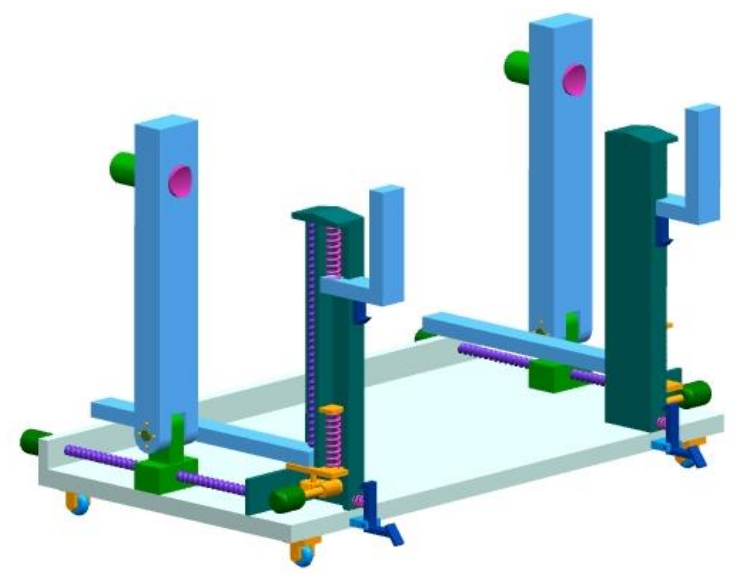

Fig.2 A new type of structural drawing of the pipe fitting rack with the function of automatic adjustment

\section{Introduction to work process}

\subsection{Composition of adjustment mechanism}

As shown in Figure 3, the adjustment mechanism of the design device is composed of fixed base, adjustment chute, adjustment slider, adjustment screw rod, adjustment motor, adjustment support plate, limit bolt, limit hole, reset clamping slot, push cylinder and push sliding plate. For the designed adjustment mechanism, two adjustment chutes are chiseled inside the fixed base, the internal sliding of the adjustment chutes is connected with two adjustment sliders, and the inner wall of the adjustment chutes is symmetrically rotated between two sides to connect the adjustment screw rods; a section of the fixed base is fixedly connected with the adjustment motor, and the output end of the adjustment motor is connected with the adjustment screw rods through the coupling; the middle part of the adjustment slider is chiseled and adjusted Adjust the screw hole of the whole screw rod engagement, adjust the screw rod to pass through the middle of the adjusting slider, adjust the top of the slider to rotate and connect the adjusting support plate; set the limit hole at the bottom of the adjusting support plate, insert the limit bolt in the middle of the limit hole; adjust the top side of the support plate to fixedly connect the push cylinder, adjust the principle of the support plate to push the cylinder, design a reset card slot, and slide the internal connection of the reset card slot to push Slide plate: the output end of the push cylinder extends into the reset card slot, and the output end is fixedly connected with the push slide plate.

Under the working condition of this design, when it is necessary to unload the pipe fittings from the transport vehicle or other places, adjust the angle of the support plate through the limit bolt and the limit hole, and roll the pipe fittings along the adjustment support plate to the top of the lifting beam in the taking mechanism. When the pipe fittings are unloaded, the adjusting screw rod is driven by the adjusting motor. Under the reaction force of the thread, the adjusting slider drives the adjusting support plate to move to the side away from the adjusting side plate. When the pressure of the adjusting support plate is reduced by the pipe fittings, the adjusting support plate is pushed to make it in the vertical state. The adjusting support plate is fixed by the limit bolt hole, and then the adjusting screw rod is driven by the adjusting motor The rod rotates in reverse direction. At this time, under the reaction force of the thread, the adjusting slider drives the adjusting support plate to move to one side of the adjusting side plate. The pipe 
fittings are arranged by pressing the pipe fittings at one time.

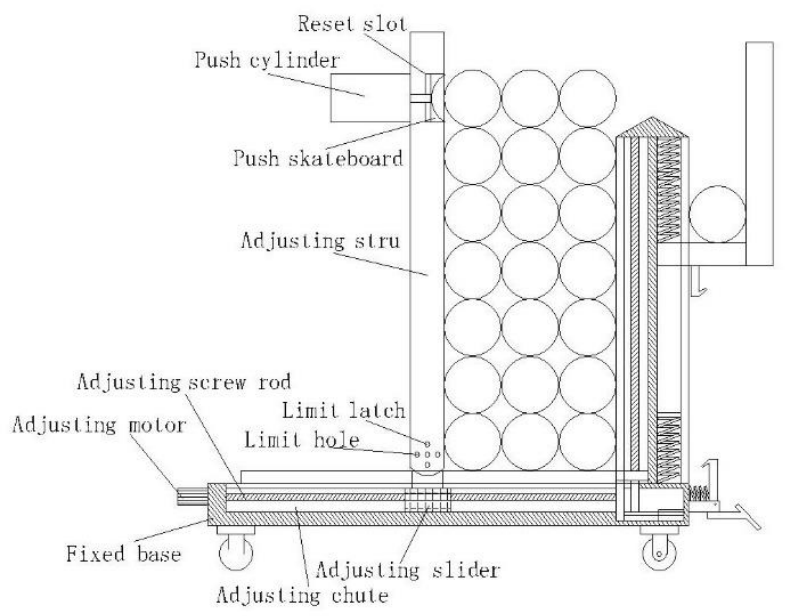

Fig.3 A new type of structure (adjusting mechanism) with automatic adjustment function

\subsection{Composition of taking mechanism}

As shown in Figure 4, the adjustment mechanism part of the design device of this project is composed of fixed base, adjustment chute, adjustment slider, adjustment screw rod, adjustment motor, adjustment support plate, limit bolt, limit hole, reset clamping groove, push cylinder and push sliding plate. For the adjustment mechanism designed in this project, two adjustment chutes are chiseled inside the fixed base, the internal sliding of the adjustment chutes is connected with two adjustment sliders, and the inner wall of the adjustment chutes is adjusted to rotate and connect the adjustment screw rods between the symmetrical sides; a section of the fixed base is fixedly connected with the adjustment motor, and the output end of the adjustment motor is connected with the adjustment screw rod through the coupling; the middle part of the adjustment slider is opened Chisel the screw hole that engages with the adjusting screw rod, adjust the screw rod to pass through the middle of the adjusting slider, adjust the top of the slider to rotate and connect the adjusting support plate; set the limit hole at the bottom of the adjusting support plate, insert the limit bolt in the middle of the limit hole; adjust the top side of the support plate to fixedly connect the push cylinder, adjust the principle of the support plate, design a reset card slot, and slide and connect the reset card slot inside The output end of the push cylinder extends into the reset card slot, and the output end is fixedly connected with the push slide plate.

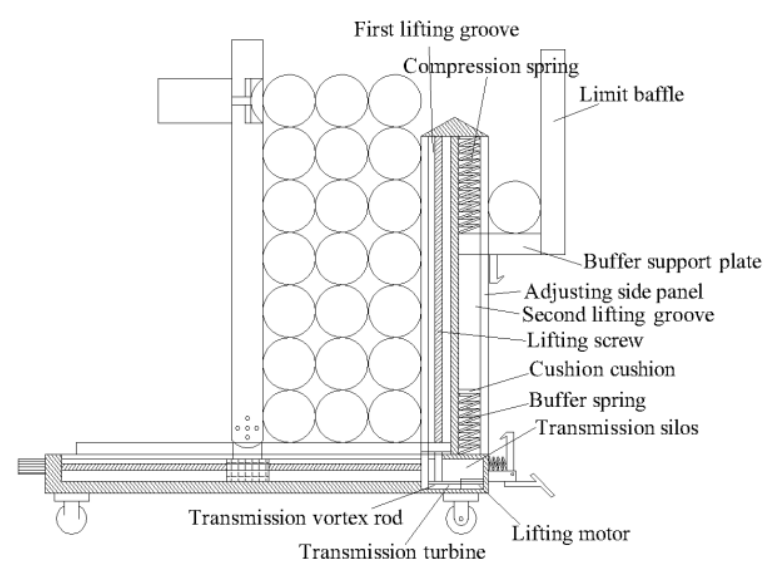

Fig.4 A new type of structural drawing (taking mechanism) of pipe fitting rack in civil engineering with automatic

Under the designed working condition, when the pipe fittings need to be removed from the transport vehicle or other places, adjust the angle of the support plate through the limit pin and the limit hole, and roll the pipe fittings along the adjustment support plate to the top of the lifting beam in the taking mechanism. When the pipe fittings are unloaded, the adjusting screw rod is driven by the adjusting motor. Under the reaction force of the thread, the adjusting slider drives the adjusting support plate to move to the side away from the adjusting side plate. When the pressure of the adjusting support plate is reduced by the pipe fittings, the adjusting support plate is pushed to make it in the vertical state. The adjusting support plate is fixed by the limit bolt hole, and then the adjusting screw rod is driven by the adjusting motor The rod rotates in reverse direction. At this time, under the reaction force of the thread, the adjusting slider drives the adjusting support plate to move to one side of the adjusting side plate. The pipe fittings are arranged by pressing the pipe fittings at one time.

\subsection{Composition of reset mechanism}

As shown in Figure 5, it is a part of the reset mechanism designed for the device of this project, which is composed of fixed support plate, foot pedal, fixed locking hook, movable locking hook and reset spring [4].

For the designed part of the reset mechanism, at the bottom of the buffer support plate in the mechanism part taken by the design device, a fixed connection movable lock hook is designed, and one end of the fixed base is located below the movable lock hook, two fixed support plates are designed to be fixedly connected, a rotation connection fixed lock hook is designed between the two fixed support plates, and a return spring is fixedly connected between the fixed lock hook and the fixed base. In the case of the design work of this project, after the operation of the take-off mechanism, under the action of the return spring, the fixed lock hook will block the movable lock hook. After the pipe fittings are taken, the foot pedal is designed to be stepped down. At this time, under the action of the compression spring, the buffer support plate is moved to the top here, so as to complete the take-off. 


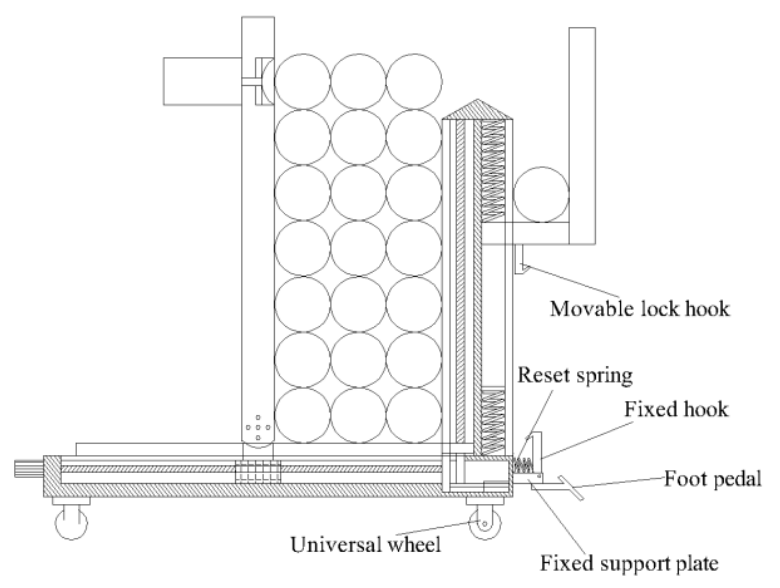

Fig.5 A new type of structure (reset mechanism) with automatic adjustment function adjustment function

The details of the worm drive part of the design device are shown in Figure 6 below. Due to the low power requirements, short single operation time and intermittent operation of the transmission mechanism in this design, the worm drive is selected as the transmission mechanism. The worm drive is mainly composed of the worm and the turbine, with the shaft intersection angle of $90^{\circ}$ to transmit the space staggered motion and power [5], for some pairs of operation When stability, compact structure, high transmission power, strong impact strength, medium and above transmission ratio, and the side clearance can be adjusted, worm drive has a very high advantage compared with its equipment [6]. In the design of this project, after the pipe fittings are arranged through the adjustment part of the device, the lifting motor drives the transmission worm wheel to rotate through the transmission worm, the transmission worm wheel drives the lifting screw rod to rotate, and the lifting screw rod drives the lifting cross beam upward through the reaction force of the thread, so the lifting cross beam lifts the pipe fittings upward.

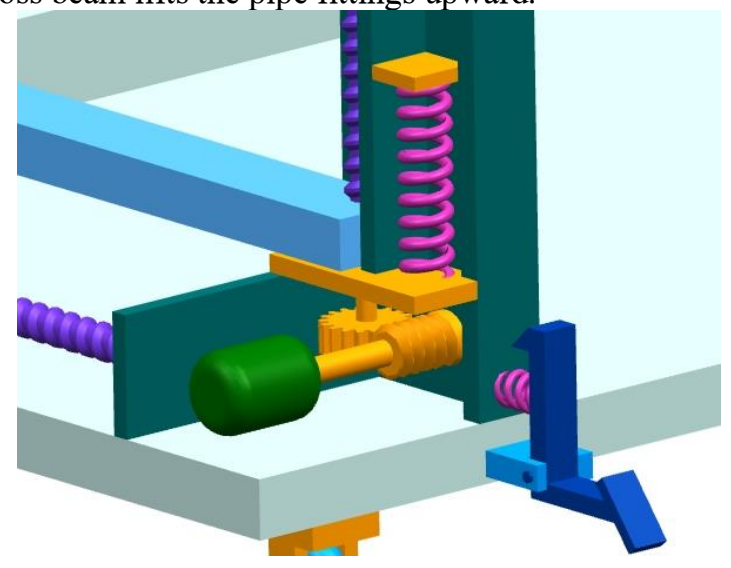

Fig.6 Worm drive structure diagram

\section{Conclusion}

In this paper, a new type of civil building pipe fitting rack is designed. According to the shortcomings of traditional pipe cone placement in the site, such as space utilization, safe and convenient handling, and reducing pipe loss, a semi-automatic equipment which can automatically adjust the pipe placement and convenient handling is researched and designed. Compared with the traditional placement, it has the following advantages:

1. Save space. The part of the device designed for this project to hold the pipe fittings is supported by the two side plates. The pipe fittings are placed from the traditional cone-shaped to the rectangular shape with the same number of pipe fittings on the upper and lower parts. In the case of occupying the same number of construction sites, more pipe fittings can be placed than the traditional way;

2. Labor saving and high efficiency. The device designed in this project has a special design. It is designed to push the pipe fittings into the buffer support plate under the action of the push cylinder through the lifting motor and buffer support plate, and send them to the bottom under the action of gravity for the use of workers, so as to realize automatic blanking and greatly improve the working efficiency of workers;

3. Easy to fix and place. The structure of the device designed in this project is compact. By setting the limit bolt and limit hole, the angle of the support rod can be adjusted flexibly through the coordination of the limit bolt and the limit hole when feeding the storage rack, so as to place the truck or high-altitude pipe fittings in the storage rack, and fix and arrange them by adjusting the motor, so as to improve the efficiency of pipe placement and reduce the labor intensity of the staff.

4. The buffer support plate, buffer spring and other buffer mechanisms designed in this project can effectively reduce the loss of pipes in the process of taking and placing, thus reducing the waste of building materials and saving the construction cost.

Through the optimization of the layout of pipe materials in the construction site, the construction site workers can be safer, the site is more orderly, the work is more efficient and the cost is more economical, which is a small step of the construction site automation, but this small step gradually accumulated has become a big step on the road of urbanization in China.

\section{References}

1. Qiuling, Chen, Zerui, et al. Solar Light Guide and Window Design Based on Energy Conservation and Renovation of Old Office Buildings[C]// 2018 International Academic Forum on architecture, aviation and environmental engineering - Internet of things. 2018.

2. Duan Hongde. How to avoid waste of construction engineering [J]. Management and technology of small and medium-sized enterprises (next ten issues), 2009 (02): 34

3. Huang Haifeng. Structural optimization design of gantry crane $[\mathrm{J}]$. Theoretical research on urban construction (electronic version), 279 (33): 95. 
4. Wang Yong, Hao Xin, Cai Zheng. Lightweight optimization design of crane turret structure [J]. Baosteel Technology, 2016 (4): 72-78.

5. Yang Renhui. Similarities and differences between screw drive and worm drive $[\mathrm{J}]$. Scientific consultation, 2015, (13): 77-77, 78. Doi: 10.3969/j.issn.1671-4822.2015.13.051.

6. Zhang Lili. Similarities and differences between screw drive and worm drive [J]. Wen Yuan (middle school edition), 2018, (11): 787. 